\title{
Vascular endothelial growth factor accelerates compensatory lung growth by increasing the alveolar units
}

\author{
Duy T Dao ${ }^{1,4}$, Prathima Nandivada ${ }^{1,4}$, Jacqueline T Vuong ${ }^{1}$, Lorenzo Anez-Bustillos ${ }^{1}$, Amy Pan ${ }^{1}$, Hiroko Kishikawa ${ }^{1}$, \\ Paul D Mitchell ${ }^{2}$, Meredith A Baker ${ }^{1}$, Gillian L Fell ${ }^{1}$, Thomas Martin ${ }^{3}$ and Mark Puder ${ }^{1}$
}

BACKGROUND: Deficiency of vascular endothelial growth factor (VEGF) is associated with hypoplastic lung diseases, such as congenital diaphragmatic hernia. Provision of VEGF has been demonstrated to be beneficial in hyperoxia-induced bronchopulmonary dysplasia, and hence could induce lung growth and improve the outcome in hypoplastic lung diseases. We aimed to determine the effects of exogenous VEGF in a rodent model of compensatory lung growth after left pneumonectomy.

METHODS: Eight-to-ten-week-old C57Bl6 male mice underwent left pneumonectomy, followed by daily intra-peritoneal injections of saline or VEGF $(0.5 \mathrm{mg} / \mathrm{kg})$. Lung volume measurement, pulmonary function tests, and morphometric analyses were performed on post-operative day (POD) 4 and 10. The pulmonary expression of angiogenic factors was analyzed by quantitative polymerase chain reaction and western blot.

RESULTS: Lung volume on POD 4 was higher in the VEGFtreated mice $(P=0.03)$. On morphometric analyses, VEGF increased the parenchymal volume $(P=0.001)$, alveolar volume $(P=0.0003)$, and alveolar number $(P<0.0001)$ on $P O D$ 4. The VEGF group displayed higher levels of phosphorylated-VEGFR2NEGFR2 $(P=0.03)$ and epidermal growth factor (EGF) messenger RNA $(P=0.01)$.

CONCLUSION: VEGF accelerated the compensatory lung growth in mice, by increasing the alveolar units. These changes may be mediated by VEGFR2 and EGF-dependent mechanisms.

A ngiogenesis plays a central role in human tissue development and regeneration. Vascular endothelial growth factor (VEGF), a key mediator of angiogenesis and vasculogenesis, is necessary for the normal development of many tissues, including the liver, brain, eyes, and lungs (1). VEGF, localized to the epithelial cell's basement membrane in the fetal lung, is thought to play a critical role in guiding the development of the newly formed capillary network within the lung (2). The absence of VEGF results in decreased lung maturation, decreased surfactant production, and hypoplasia of blood vessels and alveoli (3). These tissue abnormalities and altered levels of tissue VEGF are observed in humans and animal models exhibiting pulmonary diseases of infancy, such as respiratory distress syndrome, bronchopulmonary dysplasia, and pulmonary hypoplasia because of congenital diaphragmatic hernia (CDH) (4-7).

Our group previously demonstrated in mice that systemic administration of exogenous murine VEGF accelerated compensatory lung growth, resulting in a return to $100 \%$ of baseline lung volume in 4 days rather than 8-10 days after left pneumonectomy. The lungs in VEGF-treated animals were histologically indistinguishable from the saline-treated animals, with respect to the vascular and the alveolar density (8). However, the mechanism by which VEGF accelerates lung regeneration after unilateral pneumonectomy and its effects on pulmonary mechanics and morphometry remain unknown. The purpose of our current study was to determine the effects of systemic exogenous murine VEGF on pulmonary mechanics in a murine model of compensatory lung growth, after left pneumonectomy. We also aimed to investigate the potential cellular mechanisms, by which these changes are mediated.

\section{METHODS}

Experimental Groups and Surgical Procedure

All procedures were carried out according to the National Institutes of Health Guide for the Care and Use of Laboratory Animals and approved by the Institutional Animal Care and Use Committee at Boston Children's Hospital. Eight-to-ten-week-old C57BL/6 male mice (Jackson Laboratories, Bar Harbor, ME) were randomized into control or VEGF experimental groups. Before left pneumonectomy, the VEGF group received $0.5 \mathrm{mg} / \mathrm{kg}$ of recombinant murine VEGF $_{164}$ (GenScript, Piscataway, NJ) through intra-peritoneal (IP) injection. Control group received an isovolumetric volume of normal saline through IP injection. Mice were then anesthetized with 120$400 \mathrm{mg} / \mathrm{kg}$ of Avertin (Sigma, St. Louis, MO) using IP injection and orotracheally intubated, as previously described $(9,10)$. The animal was ventilated on room air with a rodent ventilator (HSE-HA Minivent, Harvard Apparatus, Holliston, MA) at 150 breaths/min.

\footnotetext{
${ }^{1}$ Vascular Biology Program and Department of Surgery, Boston Children's Hospital, Boston, Massachusetts; ${ }^{2}$ Institutional Centers for Clinical and Translational Research, Boston Children's Hospital, Boston, Massachusetts; ${ }^{3}$ Pulmonary Function Laboratory, Department of Medicine, Boston Children's Hospital, Boston, Massachusetts. Correspondence: Mark Puder (Mark.Puder@childrens.harvard.edu)

${ }^{4}$ These authors contributed equally to this work.

Received 18 September 2017; accepted 21 February 2018; advance online publication 11 April 2018. doi:10.1038/pr.2018.41
} 
Pneumonectomy then proceeded, as previously described (11). Postoperative pain control was achieved with twice daily subcutaneous injection of buprenorphine for $72 \mathrm{~h}$. Postoperatively, the VEGF and the control groups were further divided into two treatment-duration groups, receiving daily IP injections of VEGF or saline for either 4 or 10 days postoperatively. These two time points were chosen to compare the effects of the two treatments during (day 4) and at the end (day 10) of the proliferative phase of compensatory lung growth.

\section{Pulmonary Mechanical Studies}

On post-operative day (POD) 4 or 10 and immediately before euthanasia, animals underwent pulmonary mechanical measurements with the Flexivent system (SCIREQ, Montreal, Canada). Mice were anesthetized with Avertin, as previously described, followed by a tracheotomy and insertion of a 20-gauge hollow-bore needle, which was connected to the Flexivent system. Elastance and compliance were measured with the single-frequency forced oscillation technique, which registered the animal's response to an applied sinusoidal waveform. The system also generated pressure-volume (PV) loops, which allowed calculations of inspiratory capacity and area under the PV loop, an estimate of collapsed alveolar units before the PV loop maneuver. To normalize these parameters as per the animal's size, the inspiratory capacity, area under the PV loop, and compliance were divided by the body weight.

\section{Organ Harvest, Mass, and Volume Measurement}

Animals were euthanized upon disconnection from the Flexivent system using carbon dioxide. Animals were weighed, and the remaining right lung was removed en bloc with the tracheobronchial tree. Specimens were divided into the following two groups: morphometric analysis group and $\mathrm{RNA}$ /protein analysis group. Specimens destined for morphometric analysis were inflated with $10 \%$ formalin at $25 \mathrm{~cm} \mathrm{H}_{2} \mathrm{O}$ through the previously intubated 20 gauge needle. The trachea was ligated with a 4-0 silk suture to maintain alveolar distension, and total lung volume was measured with the water displacement method (12). The specimen was placed in $10 \%$ formalin overnight at $4{ }^{\circ} \mathrm{C}$. The liver, spleen, and kidneys were also harvested and weighed before being preserved in $10 \%$ formalin overnight at $4{ }^{\circ} \mathrm{C}$. All specimens were transferred into $70 \%$ ethanol after $24 \mathrm{~h}$ of formalin fixation, and subsequently embedded in paraffin for histologic analyses. Lung volume and organ mass measurements were normalized for body weight. Lung specimens for RNA/protein analysis were immediately flash frozen in liquid nitrogen for future tissue analysis without undergoing volume measurement.

\section{Morphometric Analysis}

Quantitative microscopy was performed on hematoxylin and eosin (H\&E)-stained lung sections, based on the principles of stereology. Five lung specimens from each group were randomly selected for morphometric analyses. One $5-\mu$ section from each specimen was examined at $\times 200$ magnification using the principle of systemic uniform random sampling: the first lung field on each section was selected randomly, followed by systemic sampling of every other field to achieve a total of 40-50 lung fields per section. For each field, a 42-point test lattice with predefined grid line length was used to facilitate the point and intersection counting $(13,14)$. Parenchyma was defined as the gas-exchange compartment of the lung, which included alveoli, terminal ducts, and alveolar septa. All volume and area measurements were normalized for body weight.

Under the assumptions that alveolar units assume a spherical shape and are randomly distributed throughout the lung parenchyma, alveolar density was mathematically calculated from counting the number of alveolar transections on randomly chosen lung fields, using the method of Weibel and Gomez. (15)

\section{Quantitative Real-Time Polymerase Chain Reaction}

Total RNA was extracted from the lung tissues with the RNeasy Mini Kit (Qiagen, Hilden, Germany), according to the manufacturer's protocol. Amplification reactions were performed, with the StepOne Real-Time PCR Systems (Applied Biosystems, Foster City, CA), using pre-designed and validated TaqMan primers for VEGF receptor (VEGFR) 1, VEGFR2, neuropilin (NRP) 1, VEGF-A, epidermal growth factor (EGF), and EGF receptor (EGFR) (Applied Biosystems). All target genes were normalized to the housekeeping gene GAPDH, and their messenger RNA (mRNA) levels were calculated using the $2^{\Delta \Delta \mathrm{Ct}}$ method (16).

\section{Western Blot and Enzyme-Linked Immunosorbent Assay}

Lung tissue samples from the animals euthanized on POD 4 were immediately suspended in radioimmunoprecipitation assay (RIPA) buffer (Boston Bio Products, Ashland, MA), containing protease and phosphatase inhibitors (Thermo Fisher Scientific, Waltham, MA). Tissue samples were then homogenized and centrifuged for $15 \mathrm{~min}$ at $4{ }^{\circ} \mathrm{C}$ and $14,000 \mathrm{rpm}$. The supernatants were collected, and the protein concentration was measured with the Bradford colorimetric assay (Bio-Rad Laboratories, Hercules, CA). Forty micrograms of protein from each sample were mixed with $4 \times$ Laemmli buffer (Boston Bio Products), heated at $95^{\circ} \mathrm{C}$ for $5 \mathrm{~min}$, and separated on a $4-12 \%$ Bis-Tris polyacrylamide gel (Thermo Fisher Scientific). The proteins were then transferred to a nitrocellulose membrane and blocked in a TBST solution $(50 \mathrm{mM}$ Tris-HCl- $\mathrm{pH} 7.4,150 \mathrm{mM}$ $\mathrm{NaCl}$, and $0.1 \%$ Tween 20 ), containing $5 \%$ non-fat dry milk (Bio-Rad Laboratories, Hercules, CA) for $1 \mathrm{~h}$. Incubation with primary antibodies at 1:1000 dilution in blocking buffer was done overnight at $4{ }^{\circ} \mathrm{C}$. Primary antibodies included anti-VEGFR1, -MMP14 (Abcam, Cambridge, UK), -VEGFR2, -P-1175-VEGFR2, -NRP1, -EGFR, -P-1068-EGFR (Cell Signaling Technology, Danvers, MA), -HB-EGF (R\&D Systems, Minneapolis, MN), and - $\beta$-Actin (SigmaAldrich, St. Louis, MO). On the following day, the membrane was washed with TBST for 10 min $3 \times$ before incubation in secondary antibodies, horseradish peroxidase-conjugated goat anti-rabbit, antimouse (Santa Cruz Biotechnology, Dallas, TX), or anti-rat IgG (R\&D Systems) antibodies at 1:2,000 dilution for $1 \mathrm{~h}$ at room temperature. The membrane was again washed with TBST, followed by signal visualization with the enhanced chemiluminescence (ECL) reagents (Thermo Fisher Scientific).

Lung tissue levels of EGF were determined with enzyme-linked immunosorbent assay (ELISA) (R\&D Systems), according to the manufacturer's protocol. Briefly, the lung tissue extract was added to the supplied ELISA plate and incubated for $2 \mathrm{~h}$ at room temperature. This was followed by horseradish peroxidase conjugation and the addition of chemiluminescent reagent. Chemiluminescent signal was detected at $450 \mathrm{~nm}$, and protein concentration was determined from a standard curve.

\section{Immunohistochemistry}

Formalin-fixed, paraffin-embedded lung sections were dried in an oven at $58{ }^{\circ} \mathrm{C}$ for $30 \mathrm{~min}$ before deparaffinization. All sections were deparaffinized using two washes of xylene, and were rehydrated using progressively lower concentrations of ethanol, ending in phosphate-buffered solution. For Ki67 staining, antigen retrieval was performed by heating the slides in a bath of citrate-based unmasking solution (Vector Laboratories, Burlingame, CA) to $120^{\circ} \mathrm{C}$ for $1 \mathrm{~min}$ in a pressurized heater (Decloaking Chamber Biocare Medical, Pacheco, CA). After cooling, the slides were incubated for $5 \mathrm{~min}$ at room temperature with $1 \%$ Triton-X. For vascular endothelium (VE)-Cadherin staining, antigen retrieval was achieved with incubation in Tris/EDTA $(\mathrm{pH}=9)$ for $5 \mathrm{~min}$ at $95^{\circ} \mathrm{C}$. Subsequently, a $3 \%$ hydrogen peroxide in methanol solution was applied for $12 \mathrm{~min}$ at room temperature to block the endogenous peroxidase. Non-specific binding was blocked with TNB (Tris $\mathrm{HCl}, \mathrm{NaCl}$, and bovine serum albumin) for $30 \mathrm{~min}$. Slides were incubated with rabbit anti-Ki67 antibody (Abcam, Cambridge, MA) in a 1:100 dilution in TNB for $3 \mathrm{~h}$ at room temperature, or goat anti-VE-Cadherin antibody (R\&D Systems) at 1:200 dilution overnight at $4{ }^{\circ} \mathrm{C}$. After washing with phosphate-buffered solution, samples were incubated with an antirabbit horseradish peroxidase-labeled polymer solution (Dako North 


\section{Articles | Dao et al.}
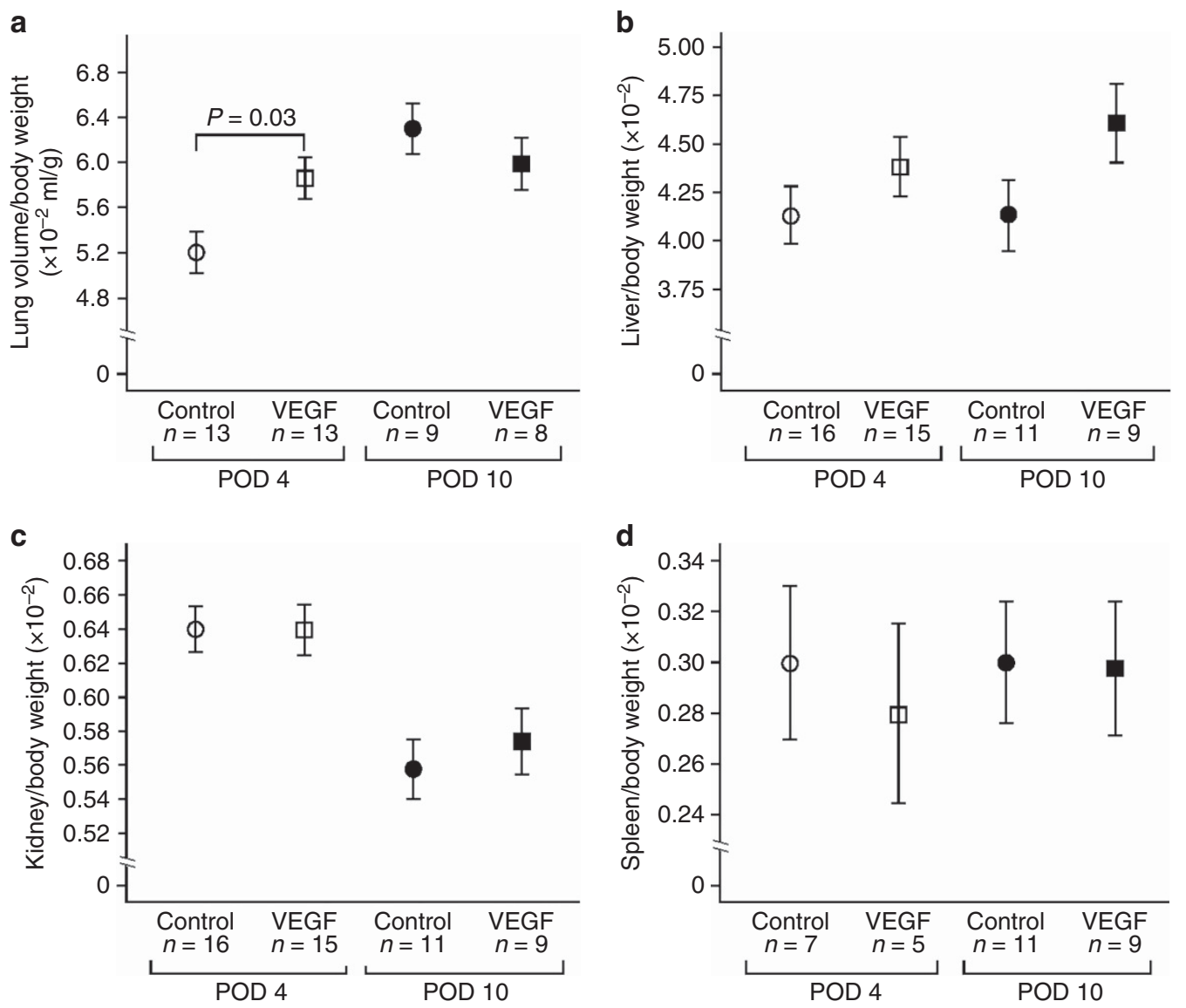

Figure 1. Organ volume and weight measurements. Mice treated with VEGF demonstrate increased lung volume/body weight ratio on postoperative day (POD) 4 (a). There is no difference between the two groups in liver (b), kidney (c), or spleen weight (d). Data are shown as mean $\pm \mathrm{SE}$.

America, Carpinteria, CA) or rabbit anti-goat IgG antibody (Vector Laboratories) for $1 \mathrm{~h}$ at room temperature. Signal visualization was achieved with diaminobenzidine (ImmPACT DAB, Vector Laboratories), followed by counterstaining with hematoxylin (Mayer's hematoxylin, Thermo Fisher Scientific). Samples were then dried overnight and mounted with xylene and Permount (Thermo Fisher Scientific).

Ki67 index was defined as the ratio between the number of Ki67positive cells (excluding alveolar macrophages) and the total number of cells. Fifteen non-overlapping random parenchymal lung fields, examined at $\times 400$ magnification, were used to calculate the average Ki67 index for each specimen. Ki67 index was estimated for lung specimens harvested on POD 2 and 4.

\section{Statistical Analysis}

Outcomes, adjusted for body weight as described above, were analyzed with a two-way analysis of variance containing a main effect for POD, a main effect for group, and the interaction effect POD $\times$ group. The interaction effect comprised six pairwise comparisons, two of which were considered a priori to be of interest (POD 4, control vs. POD 4, VEGF; POD 10, control vs. POD 10, VEGF). These comparisons were adjusted for multiplicity, using a step-up Bonferroni adjustment (17). All outcomes were normally distributed, except inspiratory capacity, parenchymal volume, alveolar volume, and mean septal thickness. For these, a sensitivity analysis was conducted by transforming each outcome using normal scores, and comparing the results to the non-transformed outcomes (18). In all cases, the results were consistent and presented in their original units. In addition, an outlier for EGF on POD 4 was shown to greatly increase the fold difference for VEGF, compared with control, so the results are reported both with and without the outlier. Tests of significance were two-sided with $P<0.05$, and considered statistically significant. Results are presented as mean \pm standard error (SE). All data analysis was conducted with SAS v9.4 (Cary, NC).

\section{RESULTS}

\section{Lung Volume and Organ Mass Measurements}

Mice in the VEGF group had a significantly higher lung volume to body weight ratio (LV/BW) on POD 4, compared with those in the control group $\left(5.86 \pm 0.18 \times 10^{-2} \mathrm{ml} / \mathrm{g}\right.$ vs. $\left.5.20 \pm 0.18 \times 10^{-2} \mathrm{ml} / \mathrm{g}, P=0.03\right)$. On POD 10 , there was no difference in LV/BW between the two groups. When normalized for body weight, there was no difference in liver, kidney, or spleen weights between the two experimental groups at either time points (Figure 1).

\section{Pulmonary Mechanical Studies}

Normalized for body weight, there was no difference between the two treatment groups at either time points (PODs 4 and 10) in inspiratory capacity or dynamic compliance (Figure 2a, c). Although not reaching the statistical significance, VEGFtreated mice on POD 4 displayed higher area under the PV loop $(P=0.1)$ (Figure 2b), an indirect measurement of 

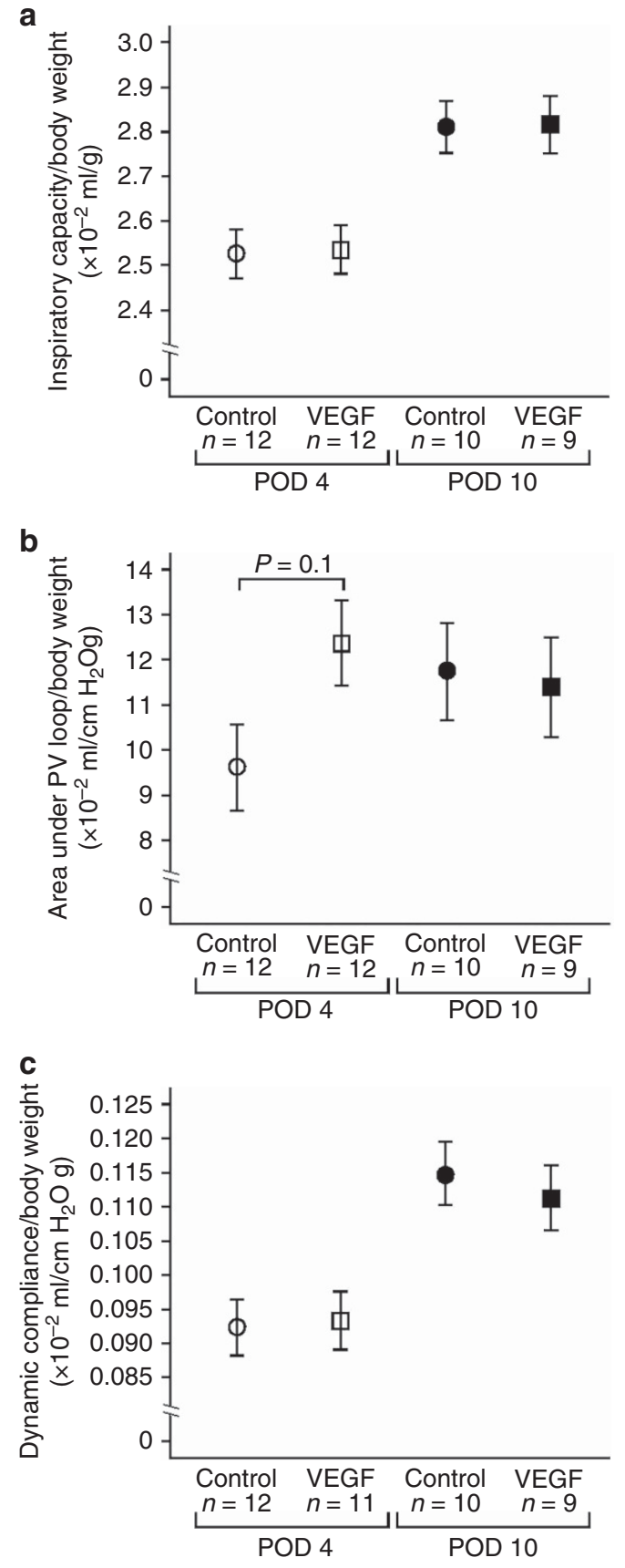

Figure 2. Pulmonary function studies. There is no difference between the two groups in normalized inspiratory capacity (a). Although not reaching statistical significance, area under the PV loop is higher in the VEGF group on POD 4 (b). There is no difference in pulmonary compliance (c). Data are shown as mean $\pm \mathrm{SE}$.

alveolar units that were available for recruitment before the PV loop maneuvers.

\section{Morphometric Studies}

Mice in the VEGF group displayed a significant increase in the adjusted parenchymal volume $\left(4.7 \pm 0.2 \times 10^{-2} \mathrm{ml} / \mathrm{g}\right.$ vs. $\left.3.8 \pm 0.2 \times 10^{-2} \mathrm{ml} / \mathrm{g}, \quad P=0.001\right), \quad$ alveolar volume $(3.0 \pm$ $0.1 \times 10^{-2} \mathrm{ml} / \mathrm{g}$ vs. $\left.2.3 \pm 0.1 \times 10^{-2} \mathrm{ml} / \mathrm{g}, \quad P=0.0003\right)$, total alveolar count $\left(4.7 \pm 0.2 \times 10^{7}\right.$ vs. $\left.2.8 \pm 0.2 \times 10^{7}, P<0.0001\right)$ and septal surface area $\left(19.0 \pm 0.7 \mathrm{~cm}^{2} / \mathrm{g}\right.$ vs. $15.5 \pm 0.7 \mathrm{~cm}^{2} / \mathrm{g}$, $P=0.007)$ on POD 4, compared with the control group. On POD 10, all of these parameters equalized; except for alveolar count, which remained higher in the VEGF group $\left(3.9 \pm 0.2 \times 10^{7}\right.$ vs. $\left.3.1 \pm 0.2 \times 10^{7}, P=0.006\right)$. Interestingly, compared with POD 4, mice treated with VEGF had a lower alveolar count on POD $10(P=0.006)$. Mice in the VEGF group had higher adjusted septal volume, compared with those in the control group on both POD $4(0.90 \pm$ $0.05 \times 10^{-2} \mathrm{ml} / \mathrm{g}$ vs. $\left.0.76 \pm 0.05 \times 10^{-2} \mathrm{ml} / \mathrm{g}, P=0.06\right)$ and POD $10\left(1.00 \pm 0.05 \times 10^{-2} \mathrm{ml} / \mathrm{g}\right.$ vs. $0.85 \pm 0.05 \times 10^{-2} \mathrm{ml} / \mathrm{g}$, $P=0.06$ ), but these differences did not meet the established statistical cutoff of $P<0.05$. There was no difference in mean septal thickness between the two groups at POD 4, but a nonsignificant elevation in the VEGF group was observed on POD $10(P=0.10$; Figure 3$)$.

\section{Gene and Protein Expression Analysis}

There was no difference in the mRNA level of VEGF, VEGFR1, VEGFR2, NRP1, or EGFR between the VEGF groups and control groups. However, VEGF-treated mice displayed a 20.5-fold increase in the level of EGF on POD 4, compared with the control group; even after omission of one outlier among the VEGF group, there remained a statistically significant five-fold increase $(P=0.02)$ (Figure 4a). Efforts to determine the protein levels of EGF were unsuccessful, since ELISA failed to detect its presence in lung tissue. There was no difference in protein expression levels of VEGFR1 or NRP1 between the two experimental groups. However, the activation level of VEGFR2 on POD 4 was significantly higher in the VEGF group, compared with the control group $(P=0.03)$ (Figure $\mathbf{4 b}, \mathbf{c})$. Although not reaching statistical significance, the activation level of EGFR was also higher in the VEGF group $(P=0.09)$. There was no difference in the expression levels of matrix-metalloproteinase (MMP14) and heparin-binding epidermal growth factor (HB-EGF) between the two groups.

\section{Immunohistochemistry}

Immunohistochemistry (IHC) labeling of lung sections for VE-Cadherin revealed uniform staining of alveolar septa in both groups (Figure 5a). The Ki67 index was significantly higher in the VEGF group on POD 2, as compared with the control group $(0.18 \pm 0.02$ vs. $0.11 \pm 0.02, P=0.02)$ (Figure 5b). However, this relationship reversed on POD 4, when more proliferation was seen in the control group $(0.25 \pm 0.02$ vs. $0.16 \pm 0.03, P=0.02)$ (Figure 5c). These findings suggested a shift toward earlier cellular proliferation, as a result of systemic VEGF treatment.

\section{DISCUSSION}

The need for therapies to accelerate lung development in infants with pulmonary disease continues to be of high priority. Exogenous VEGF administration and VEGF-gene therapy have been investigated in animal models exhibiting 

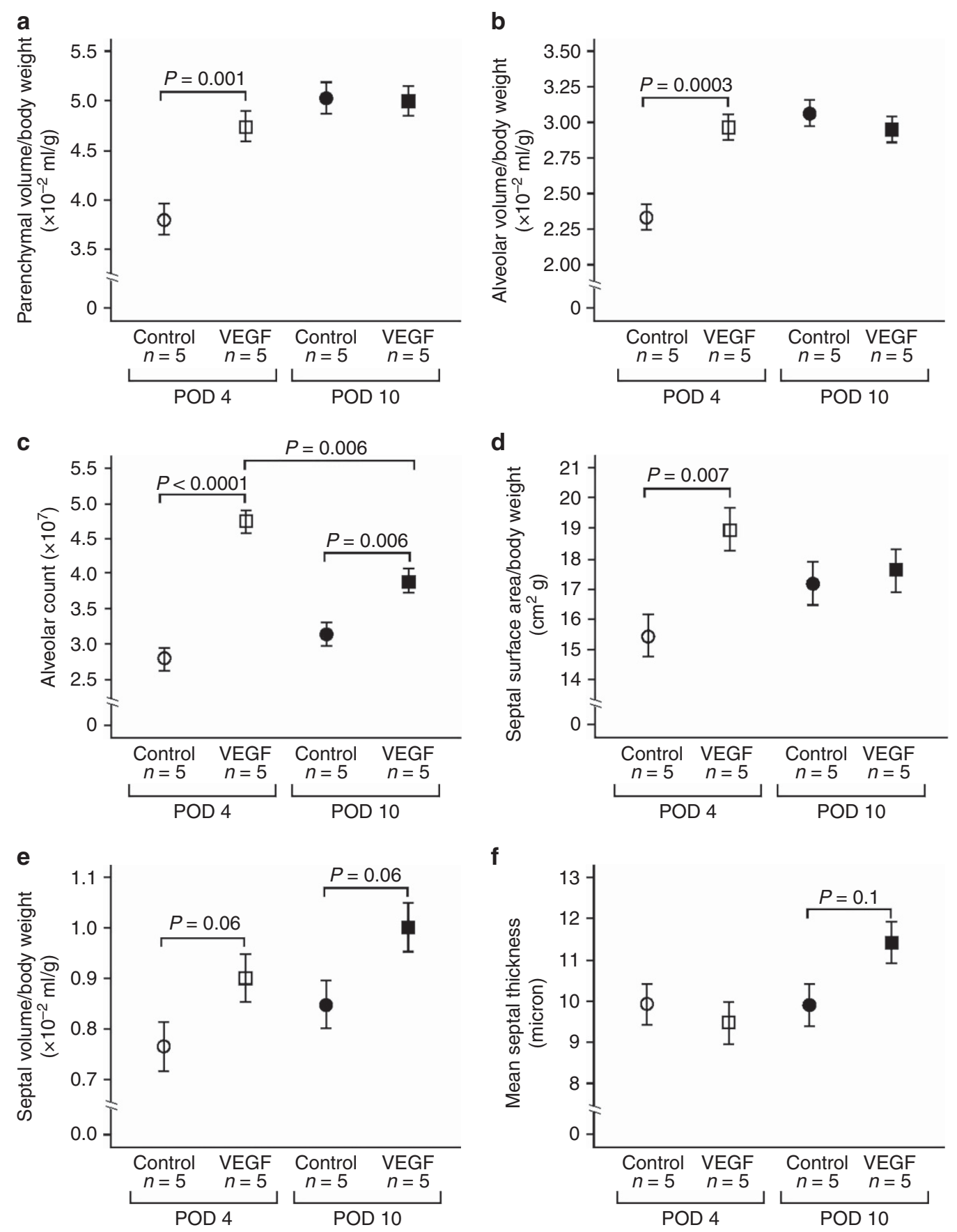

Figure 3. Morphometric analyses. VEGF-treated mice display increased parenchymal volume (a), alveolar volume (b), and septal surface area (d) on post-operative day (POD) 4. Alveolar count is higher in the VEGF group on both POD 4 and 10 (c), but there is a decrease in alveolar count in the VEGF group from POD 4 to 10. There is a trend toward significance for higher septal volume on POD 4 and 10 in the VEGF group (e). There is no statistical difference in mean septal thickness between the two groups, although the value is higher on POD 10 with VEGF treatment. Data are shown as mean $\pm \mathrm{SE}$.

respiratory distress syndrome and bronchopulmonary dysplasia with evidence of improved capillary and alveolar development, supporting the hypothesis that postnatal VEGF therapy may be a viable option $(3,7)$.

We previously demonstrated that administration of systemic exogenous murine VEGF accelerated the compensatory lung growth in 8-week-old mice after left pneumonectomy, with the preservation of normal pulmonary architecture (8). Our current study demonstrated that there were no differences in several measures of pulmonary mechanics between mice that received VEGF and those that received saline after left pneumonectomy, despite of an increased total lung volume in the VEGF-treated group. Specifically, VEGF therapy did not alter the dynamic compliance or resistance, 

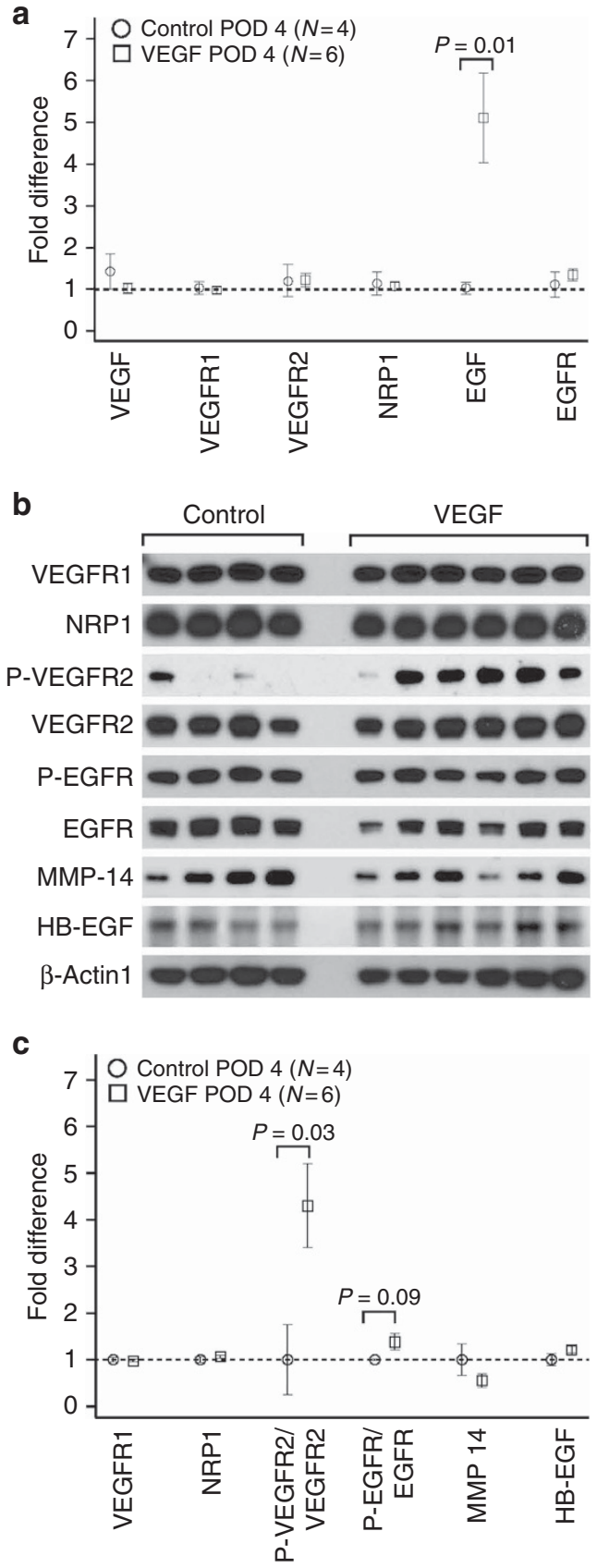

Figure 4. Gene and protein expression analyses. VEGF-treated lungs on post-operative day (POD) 4 display increased messenger RNA (mRNA) expression of epidermal growth factor (EGF) on quantitative polymerase chain reactions (a). VEGF-treated lungs also display increased VEGFR2 activation on western blot (b, c). Although not reaching statistical significance, there is also an increase in EGFR activation (b, c). Data are shown as mean $\pm \mathrm{SE}$.

suggesting that the tissue changes that occurred in the VEGFtreated group did not alter the overall mechanics. It should also be noted that despite a small and statistically insignificant increase in mean septal thickness in the VEGF group on POD 10, which was likely a result of an increased vascular permeability associated with prolonged systemic administration, there were no changes in the pulmonary mechanics.
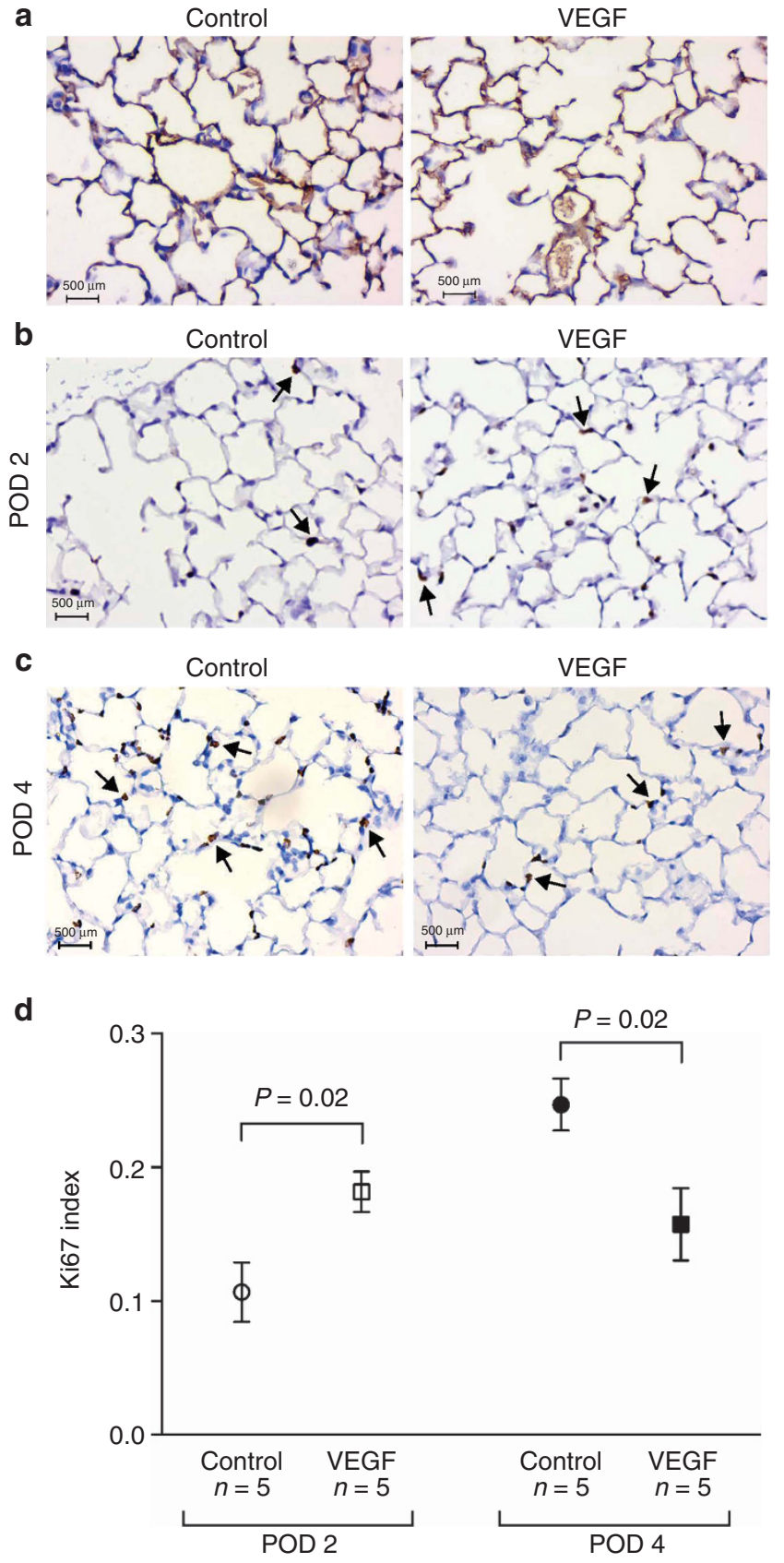

Figure 5. Immunohistochemistry. VE-Cadherin-labeled lung sections reveal no difference in pulmonary vasculature between the two treatment groups (a). On Ki67 staining, cellular proliferation increased with VEGF treatment on POD 2, but reduced on POD 4 (b-d). Data for $\mathrm{Ki} 67$ index are shown as mean $\pm \mathrm{SE}$.

These findings are relevant, since a previous report demonstrated that treatment of the lung primordia from mice with and without exposure to nitrofen, and the subsequent treatment with fibroblast growth factor (FGF) resulted in cystic changes within the lung (19). There was also no difference in inspiratory capacity between VEGF-treated and saline-treated mice, despite increased total lung volume, as 


\section{Articles | Dao et al.}

measured by volume displacement in VEGF-treated mice. It is possible that additional alveolar recruitment maneuvers are necessary to reflect the increased lung volume observed in the VEGF-treated group.

On morphometric analyses, VEGF-treated mice displayed both increased alveolar volume and septal surface area, on POD 4. Most importantly, VEGF treatment resulted in an increase in the total alveolar count, an effect that persisted even on POD 10. Interestingly, the alveolar count decreased from POD 4 to POD 10 in the VEGF group, which may represent a resorption of immature alveoli created in the early phase of VEGF treatment. In adult mice, a decrease in alveolar count without changes in lung volume has been observed over the course of lung development (20). VEGF treatment seemed to replicate this process, albeit within a much shorter duration. In addition, IHC labeling of lung sections with VE-Cadherin showed uniform staining of alveolar septa, suggesting that the increase in alveolar units and septal surface area was accompanied by an increase in vascularization, and that VEGF administration led to an increase in the total capillary volume.

Our data also demonstrated that the observed volumetric, mechanical, and morphometric findings were associated with measurable changes in gene and protein expression. The activated state of pulmonary VEGFR2 was significantly increased by exogenous VEGF therapy after unilateral pneumonectomy in our study. Ding et al. similarly observed that the process of compensatory lung growth was highly dependent on VEGFR2, with the proliferation of both pulmonary capillary endothelial cells and type II pneumocytes significantly impaired in endothelial-specific VEGFR2 knockout mice (21). This group also demonstrated that the proliferation of pulmonary epithelial cells is dependent on the endothelial cell-derived growth factors that act in a paracrine fashion. Based on our findings, it is possible that exogenous VEGF accelerates the compensatory lung growth by directly stimulating pulmonary capillary endothelial cell proliferation through a VEGFR2-dependent mechanism, which subsequently induces alveolar epithelial cell growth and formation of new alveolar units. IHC analyses, in fact confirmed increased cellular proliferation on POD 2 with VEGF treatment, as reflected by a higher Ki67 index. It should be noted that this relationship of Ki67 index was reversed on POD 4, when cellular proliferation was higher in the control group. These findings are consistent with our conclusion that VEGF accelerated the compensatory lung growth. With exogenous VEGF, the proliferative phase of lung growth was largely finished by POD 4, leading to a decrease in the Ki67 index.

It should also be noted that in a previous study by our group, the administration of a VEGFR2 inhibitor, in the form of an antibody (DC101) or small chemical inhibitor (SU11248), did not result in measurable changes in lung growth (8). There could be two explanations for this observation. First, other angiogenic growth factors could likely contribute to the compensatory lung growth and compensate for VEGF in the face of its receptor's blockade. Second, these agents might not have provided sufficient blockade of VEGFR2 when administered at the provided doses, and hence, a dose-response study could have provided different results.

This study also demonstrated a significant increase in the expression of EGF mRNA in VEGF-treated mice after unilateral pneumonectomy. ELISA, however, failed to detect EGF levels in lung tissues, probably because of its small size, secretory nature, and lack of interaction with the extracellular matrix that contributed to its low local concentrations (22). Although not reaching statistical significance, there was also an increase in the activation of EGFR, supporting the finding of increased EGF transcription. Ding et al. reported that activation of VEGFR2 resulted in an upregulation of MMP14, which in turn released EGF-like ligands, such as HB-EGF, and induced alveolar epithelial cell proliferation (21). Interestingly, in our study, treatment with VEGF did not alter the levels of MMP14 or HB-EGF, suggesting that exogenous VEGF upregulated EGF in a mechanism that is separate from the natural compensatory lung growth.

A major limitation of this study was the lack of equivalency between the left pneumonectomy model, which was done on healthy lungs, and pulmonary hypoplasia associated with $\mathrm{CDH}$. Another limitation was the use of a compensatory lung growth model to simulate the process of lung development. Although lung development is distinctly different from compensatory lung growth after pneumonectomy, there are fundamental commonalities between these two processes. They are both driven by mechanical stress that is created by either a symmetrically enlarging thorax (lung development) or by an asymmetrical empty space (compensatory growth), with the hypoxia-induced factor (HIF)-VEGF axis playing a critical role in both models (23). In addition, at the level of gene transcription, post-pneumonectomy lungs have been shown to first dedifferentiate before undergoing redevelopment, a process that recapitulates the late alveolar stage of lung development (24).

\section{ACKNOWLEDGMENTS}

We acknowledge Kristin Johnson of the Vascular Biology Program at Boston Children's Hospital for her work in preparation of the figures.

\section{STATEMENT OF FINANCIAL SUPPORT}

Research funding for this study was provided by the Boston Children's Hospital Surgical Foundation and the Vascular Biology Program of Boston Children's Hospital (D.T.D., L.A.-B., A.P., M.A.B., G.L.F., and M.P.), Shire/ Boston Children's Hospital Rare Disease Collaboration (P.N., D.T.D.), the Joshua Ryan Rappaport Fellowship (P.N.), the Corkin and Maher Family Fund (P.D.M.), and the National Institutes of Health Grants 5T32HL007734 (D.T.D., M.A.B.), 4T35HL110843 (J.T.V.), and 1F32DK104525-01 (G.L.F.).

Disclosure: The authors declare no conflict of interest that could affect their work or conclusions of this study.

\section{REFERENCES}

1. Carmeliet P. Angiogenesis in life, disease and medicine. Nature 2005;438: 932-6. 
2. Acarregui MJ, Penisten ST, Goss KL, Ramirez K, Snyder JM. Vascular endothelial growth factor gene expression in human fetal lung in vitro. Am J Respir Cell Mol Biol 1999;20:14-23.

3. Compernolle V, Brusselmans K, Acker T, et al. Loss of HIF- $2 \alpha$ and inhibition of VEGF impair fetal lung maturation, whereas treatment with VEGF prevents fatal respiratory distress in premature mice. Nat Med 2002;8:702.

4. Chang R, Andreoli S, Ng Y-S, et al. VEGF expression is downregulated in nitrofen-induced congenital diaphragmatic hernia. J Pediatr Surg 2004;39: 825-8.

5. Lassus P, Ristimaki A, Ylikorkala O, Viinikka L, Andersson S. Vascular endothelial growth factor in human preterm lung. Am J Respir Crit Care Med 1999;159:1429-33.

6. Shehata SMK, Mooi WJ, Okazaki T, El-Banna I, Sharma HS, Tibboel D. Enhanced expression of vascular endothelial growth factor in lungs of newborn infants with congenital diaphragmatic hernia and pulmonary hypertension. Thorax 1999;54:427-31.

7. Thébaud B, Ladha F, Michelakis ED, et al. Vascular endothelial growth factor gene therapy increases survival, promotes lung angiogenesis, and prevents alveolar damage in hyperoxia-induced lung injury: evidence that angiogenesis participates in alveolarization. Circulation 2005;112: 2477-86.

8. Sakurai MK, Lee S, Arsenault DA, et al. Vascular endothelial growth factor accelerates compensatory lung growth after unilateral pneumonectomy. Am J Physiol Lung Cell Mol Physiol 2007;292:L742-7.

9. Gibney BC, Houdek JP, Chamoto K, et al. Mechanostructural adaptations preceding postpneumonectomy lung growth. Exp Lung Res 2012;38: 396-405.

10. Hamacher J, Arras M, Bootz F, Weiss M, Schramm R, Moehrlen U. Microscopic wire guide-based orotracheal mouse intubation: description, evaluation and comparison with transillumination. Lab Anim 2008;42: 222-30.

11. Sakurai MK, Greene AK, Wilson J, Fauza D, Puder M. Pneumonectomy in the mouse: technique and perioperative management. J Investig Surg 2005;18:201-5.
12. Scherle W. A simple method for volumetry of organs in quantitative stereology. Mikroskopie 1970;26:57-60.

13. Mühlfeld C, Ochs M. Quantitative microscopy of the lung: a problembased approach. Part 2: stereological parameters and study designs in various diseases of the respiratory tract. Am J Physiol Lung Cell Mol Physiol 2013;305:L205-21.

14. Ochs M, Mühlfeld C. Quantitative microscopy of the lung: a problembased approach. Part 1: basic principles of lung stereology. Am J Physiol Lung Cell Mol Physiol 2013;305:L15-22.

15. Weibel ER, Gomez DM. A principle for counting tissue structures on random sections. J Appl Physiol 1962;17:343-8.

16. Livak KJ, Schmittgen TD. Analysis of relative gene expression data using real-time quantitative PCR and the $2^{\wedge}(-\Delta \Delta \mathrm{CT})$ method. Methods 2001;25:402-8.

17. Hochberg Y. A sharper Bonferroni procedure for multiple tests of significance. Biometrika 1988;75:800.

18. Ludwig O, Blom G. Gunnar: Statistical estimates and transformed betavariables. Biometrische Zeitschrift 1961;3:285.

19. Jesudason EC, Connell MG, Fernig DG, Lloyd DA, Losty PD. In vitro effects of growth factors on lung hypoplasia in a model of congenital diaphragmatic hernia. J Pediatr Surg 2000;35:914-22.

20. Pozarska A, Rodríguez-Castillo JA, Surate Solaligue DE, et al. Stereological monitoring of mouse lung alveolarization from the early postnatal period to adulthood. Am J Physiol Cell Mol Physiol 2017;312:L882-95.

21. Ding B-S, Nolan DJ, Guo P, et al. Endothelial-derived angiocrine signals induce and sustain regenerative lung alveolarization. Cell 2011;147: 539-53.

22. Carpenter G, Cohen S. Epidermal growth factor. J Biol Chem 1990;265: 7709-12.

23. Hsia CCW. Comparative analysis of the mechanical signals in lung development and compensatory growth. Cell Tissue Res 2017;367: 687-705.

24. Kho AT, Liu K, Visner G, Martin T, Boudreault F. Identification of dedifferentiation and redevelopment phases during postpneumonectomy lung growth. Am J Physiol Lung Cell Mol Physiol 2013;305:L542-54. 